\title{
Rosai-Dorfman-Destombes disease: isolated intracranial without nodal involvement presentation. Illustrative case
}

\author{
César Chong, MD, ${ }^{1}$ Xavier Wong-Achi, MD, ${ }^{1,3}$ and Cinthya Apolo, $M D^{2,3}$ \\ Departments of ${ }^{1}$ Neurosurgery and ${ }^{2}$ General Surgery, National Oncologic Institute "Dr. Juan Tanca Marengo" ION-SOLCA, Guayaquil, Ecuador; and ${ }^{3}$ Universidad Espíritu \\ Santo, Samborondón, Ecuador
}

\begin{abstract}
BACKGROUND Rosai-Dorfman-Destombes disease is a rare and heterogeneous entity that has been associated with autoimmune, hereditary, and malignant diseases. There is controversy about its etiopathogenesis, clinical course, and therapeutic management.

OBSERVATIONS The authors report a case of a 61-year-old man with a history of progressive headache without any other symptoms. Magnetic resonance imaging of the brain revealed multiple irregular lesions with an initial diagnostic impression of meningiomatosis. An excisional biopsy was performed, and the pathology report stated the finding was Rosai-Dorfman-Destombes disease.

LESSONS The uniqueness of this case is its rarity. The isolated intracranial location presents many diagnostic and therapeutic challenges, with radiological and clinical characteristics similar to those of other central nervous system tumors. There is currently no clear evidence of the pathogenesis and therapeutic management of this condition. Follow-up of these patients will help elucidate the natural history of this condition and the benefits of various treatment modalities.
\end{abstract}

https://thejns.org/doi/abs/10.3171/CASE21238

KEYWORDS Rosai-Dorfman-Destombes disease; histiocytosis; central nervous system; oncology

Rosai-Dorfman-Destombes disease (RDD) is a rare condition also known as "non-Langerhans cell histiocytosis." Initially described by Pierre-Paul Destombes in 1965, it has historically been considered a benign proliferative condition of undetermined etiology, with a few patients having poor outcomes. ${ }^{1}$

Patients with a classic presentation of RDD show bilateral cervical lymphadenopathy. Up to $43 \%$ of patients have been reported to show extranodal involvement, with the head and neck region, upper respiratory tract, and skin being the most affected locations. In $<5 \%$ of cases, this condition has been reported in the central nervous system (CNS). The intracranial presentation is usually associated with nodal involvement, and isolated intracranial RDD without nodal involvement is an extremely rare condition $(0.5 \%)$, according to the literature. ${ }^{2,3}$

This is a heterogeneous entity that has been associated with autoimmune, hereditary, and malignant diseases. There is controversy about its etiopathogenesis, clinical course, and therapeutic management. Due to the nonspecific radiological signs, an initial diagnosis of meningioma is usually made, and patients undergo surgery, with a different pathological diagnosis made later.,

An evidence-based approach is necessary for the diagnostic evaluation and treatment of this disease that presents with a broad clinical spectrum. ${ }^{1}$ In this study, we present a rare case of isolated intracranial RDD without any other systemic involvement.

\section{Illustrative Case}

\section{History and Examination}

A 61-year-old man was referred with a 2-month history of headache with progression of intensity over the last 3 weeks. His past medical history included traumatic brain injury 8 years earlier without neurological sequelae. He reported no history of other symptoms. On physical examination, the patient was conscious and alert. No focal abnormalities were found on neurological examination. The findings of routine laboratory studies were unremarkable.

ABBREVIATIONS CNS = central nervous system; $C T$ = computed tomography; MRI = magnetic resonance imaging; RDD = Rosai-Dorfman-Destombes disease. INCLUDE WHEN CITING Published June 14, 2021; DOI: 10.3171/CASE21238.

SUBMITTED April 14, 2021. ACCEPTED April 20, 2021.

(c) 2021 The authors, CC BY-NC-ND 4.0 (http://creativecommons.org/licenses/by-nc-nd/4.0/). 

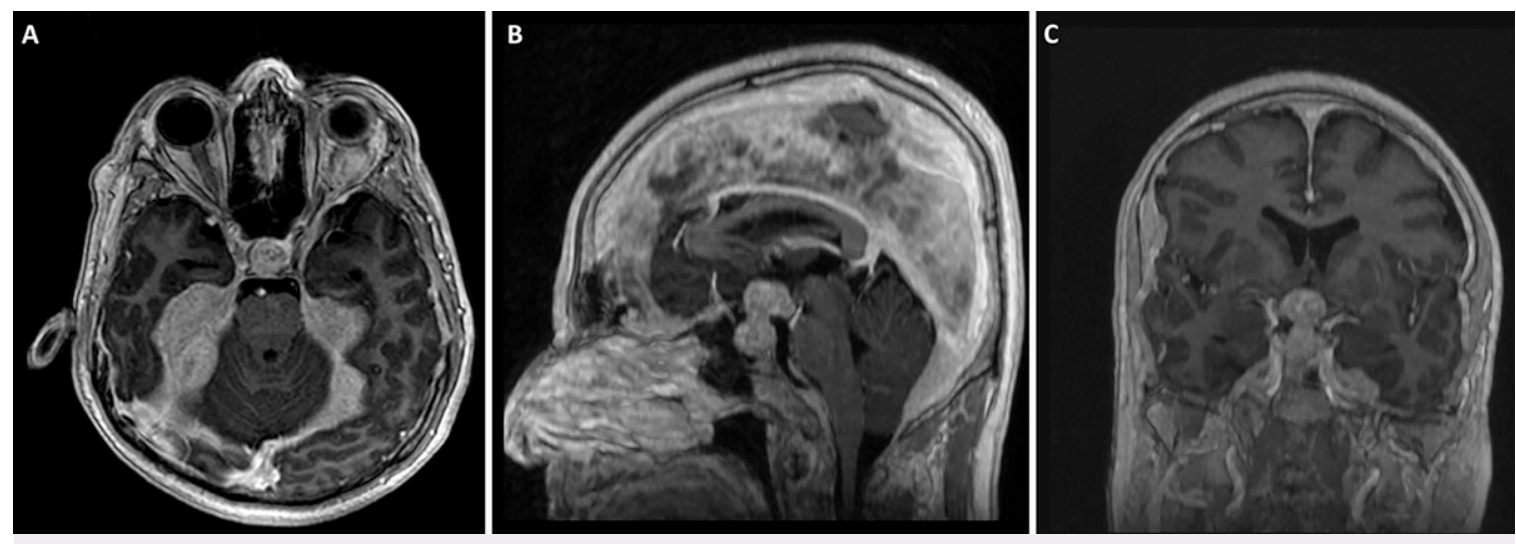

FIG. 1. Contrast-enhanced T1-weighted MRI in axial (A), sagittal (B), and coronal (C) planes showing extensive, uniformly enhancing lesions with an irregular pattern along the skull base and brain convexity, extending to the falx cerebri and tentorium cerebelli, and a sellar lesion with para- and suprasellar extension that displaces both the pituitary stem and the optic chiasm.

Contrast-enhanced magnetic resonance imaging (MRI) of the head revealed multiple irregular lesions with moderate signal enhancement located in the sellar-suprasellar region $(29.7 \times 24.9 \times$ $16.9 \mathrm{~mm}$ ) with infiltration of the pituitary stem and optic chiasm and projection toward the suprasellar cistern. Another neoformation occupied the skull base and extended through the cisternal spaces to the posterior fossa, tentorium cerebelli, and subdural space in both brain hemispheres (Fig. 1). Based on radiological findings, a differential diagnosis of meningioma was considered.

\section{Surgery and Postoperative Course}

After giving informed consent, the patient underwent a right parietal craniotomy for complete excision of one of the tumors located on the convexity. With neuronavigation assistance, the tumor volume and main vessels were established. Durotomy was performed, evidencing a low-vascularization tumor of fibrous consistency measuring $3 \mathrm{~cm}$ in diameter and firmly adhered to the meninges. Compression without infiltration of the brain tissue was observed.

Postoperatively, the patient remained without focal neurological symptoms but presented an episode of severe hypernatremia (173 $\mathrm{mEg} / \mathrm{L})$ with altered sensorium, polyuria $(5,200 \mathrm{~mL} / 24$ hours), and low cortisol upon being evaluated by the endocrinologist. Transient hypopituitarism was diagnosed, and treatment with desmopressin, hydrocortisone, and levothyroxine was initiated. After resolution of symptoms, the patient was discharged 5 days after surgery with subsequent follow-up.

\section{Histopathological Examination}

Microscopic examination of the specimen revealed a mixed inflammatory infiltrate of mature histiocytes with evidence of emperipolesis, $T$ and $\mathrm{B}$ lymphocytes, and plasma cells, showing positive staining for S100 and CD68 and negative staining for CD3 and CD20. The Ki67 index was $15 \%$. The histopathology report revealed RDD.

\section{Postoperative Follow-Up}

At his 3-month postoperative visit, the patient's mild headache persisted without any other symptoms. The patient was screened for systemic disease; a bone marrow biopsy was performed without any findings. On a full-body computed tomography (CT) scan, no lymphadenopathy or systemic masses were identified, and no neuroradiological features indicative of progression were noted. Adjuvant treatments (radioor chemotherapy) were not administered. The patient remains under observation with periodic follow-up.

\section{Discussion}

$\mathrm{RDD}$ is a rare condition whose prevalence has been described as 1 in 200,000. It is more frequently described in children and young adults and has a male sex predilection, although it has been reported in elderly patients. ${ }^{1,2}$

The etiology is not well defined. Studies have associated RDD with viral infections (herpesviruses, Epstein-Barr virus, and human immunodeficiency virus); however, a clear association has not been evidenced. Histological features of RDD have also been described in patients with myelodysplastic syndrome, Hodgkin and non-Hodgkin lymphomas, or after bone marrow transplant for acute leukemia. ${ }^{5,6}$ The association with autoimmune diseases has been described in $10 \%$ of cases. ${ }^{6}$

Due to the nonspecificity of the laboratory and imaging tests, the diagnosis of this entity is mainly histological, showing dilated lymph node sinuses with foamy histiocytes and plasma cells. The presence of histiocytes with emperipolesis (intact hematopoietic cells, mostly lymphocytes within their cytoplasm), ${ }^{2}$ although not specific, has been considered an important indicator of RDD, and it was a remarkable finding in the pathological specimen of our patient. It should be noted that in intracranial RDD, this histological finding is often unnoticed by extensive fibrosis, as described by Andriko et al. ${ }^{7}$

Immunohistochemical analysis consistently shows positive protein S100 and CD68 and CD1a negativity. ${ }^{1,2}$ The histopathological differential diagnosis should include Langerhans cell histiocytosis, lymphoplasmacytic meningioma, Hodgkin disease (nodular sclerosing variety), inflammatory pseudotumor, and plasmacytoma.

Clinically, this condition can be divided into two forms of presentation: classic nodal RDD and extranodal RDD. Patients with nodal disease present with bilateral and massive painless cervical lymphadenopathy, and extranodal disease has been reported in $43 \%$ of cases. ${ }^{1,2}$ Multisystemic involvement has been described in up to $19 \%$ of cases, and the prognosis is correlated with the number of lymph node groups and total extranodal systems involved. ${ }^{4}$

CNS involvement has been described in $<5 \%$ of cases with or without nodal involvement, with $75 \%$ occurring as intracranial lesions and $25 \%$ as spinal lesions. ${ }^{1}$ Isolated intracranial RDD 
without nodal involvement is reported as extremely rare. ${ }^{2,4,8}$ This variety of presentation has been reported in older patients and without lymphadenopathy, with a diversity of symptoms, including headache, deficits in sensory or motor systems, cranial neuropathy, seizures, or gait disturbance, usually evolving over weeks or months. ${ }^{2,9}$ Our patient complained only of headache that disturbed his daily activities.

The most frequent radiological finding of intracranial RDD is a solitary mass or multiple extraaxial dural masses, which enhance homogeneously and can be confused with meningioma or diffuse pachymeningitis.

Parenchymal lesions are rare and can be located infra- or supratentorially, intraventricularly, or multifocally. RDD involving the sellar region has been reported as rare. ${ }^{10}$ Our patient presented with an otherwise asymptomatic sellar lesion with para- and suprasellar extension; however, postoperatively, the patient presented with a transient hypopituitarism without reasonable explanation because the resected lesion was located in the hemispheric extraaxial area. This condition improves after treatment. Cerebrospinal fluid analysis may show lymphocytic pleocytosis, elevated protein with low glucose, and also emperipolesis, although it is often unremarkable. ${ }^{1,9,11,12}$ Other forms of presentation involve the nasal cavity and paranasal sinuses and the gastrointestinal, renal, and hematological systems. ${ }^{5,13-15}$

The diagnostic evaluation of patients with RDD should include the disease extent, as well as evaluation for related conditions (autoimmune disorders, viral infections, and secondary malignancies). In older patients, a full-body CT scan is recommended, ${ }^{16}$ and in patients with ocular or neurological symptoms, contrast-enhanced MRI of the head should be performed in order to identify neurological involvement. Laboratory evaluation should include a complete blood and metabolic panel; viral serology is suggested to exclude associated diagnoses. Patients with unexplained cytopenia or abnormal peripheral blood cells may require bone marrow biopsy.

A therapeutic approach for RDD has not been determined, so the treatment is adapted to the clinical condition of the patient. Because of the multiple lesions that frequently occur, the objective of surgery is limited to biopsy for pathological study; however, for unifocal lesions, complete resection can be curative. In patients with isolated intracranial disease, long-term remissions have been reported with resection alone. ${ }^{17,18}$ Due to the extension of the disease in our patient, it was decided to perform only an excisional biopsy of one of the lesions.

Steroids have become helpful in reducing node size and symptoms, although the literature reports variable response. ${ }^{1}$ Dexamethasone (8-20 mg/day) and prednisone (40-70 mg/day) are the most widely used, showing effectiveness with partial or complete response in cases with CNS involvement. However, it should be noted that there is no defined dose or duration. A feasible approach is to dose to the best observed response, followed by a gradual taper. ${ }^{19,20}$

Treatment with chemotherapy drugs has shown mixed results. Although this modality is generally reserved for refractory or relapsed cases, its use as initial therapy in disseminated or life-threatening disease has been reported. ${ }^{21,22}$ Radiation therapy has shown moderate efficacy in intracranial RDD; nonetheless, it can be beneficial in refractory disease or as palliative therapy. ${ }^{22}$ Its use has also been described in patients with symptom recurrence after surgery and when other treatments are contraindicated. According to the literature, standard doses have not been established, with doses used reported between 30 and $50 \mathrm{~Gy}^{11,23}$
Targeted therapeutic agents such as imatinib mesylate have shown some activity in RDD. The robust effectiveness of imatinib mesylate in other histiocytosis cases raises interest in their potential for RDD; however, the broad applicability of this agent in intracranial RDD has not yet been established. ${ }^{1,22}$

The optimal duration of treatment is not known, because the clinical course is unpredictable, regardless of treatment. A reasonable approach of 6-12 months of systemic therapy followed by observation has been proposed in the literature, ${ }^{1}$ and the interval of surveillance could be extended if disease stabilizes or remission occurs. ${ }^{2,22}$

There is a recent surge in the documentation of RDD in the literature, with a significant increase in the proportion of cases involving the CNS. ${ }^{24-26}$ We emphasize that RDD should be considered in the differential diagnosis of dural meningiomas.

\section{Observations}

RDD is an uncommon histiocytic disorder with two clinical forms of presentation: nodal and extranodal. No uniform approach has been determined, and treatment is best tailored to the individual clinical circumstances. Observation is reasonable in many cases; it has been described that $20-50 \%$ of patients will have spontaneous remissions. Surgery is a feasible option if the lesions are symptomatic or expanding. Surgical treatment can be challenging; lesions must be evaluated individually, and it must be taken into account which lesion should be removed initially. The mere presence of multiple lesions does not justify resection of all of them. If possible, the goal of surgery is to achieve a gross-total resection.

The data reported in the literature are insufficient to estimate the prognosis of this condition in detail. Patients with extranodal disease experience an unpredictable clinical course, with periods of remission and reactivation that may last years. In the reported patient, due to the mild symptoms and the nonprogression of the clinical or radiological state of the disease, periodic follow-up was decided, and adjuvant treatment was not prescribed.

\section{Lessons}

The uniqueness of this case is its rarity. The isolated intracranial location represents an unusual manifestation of this disease and appears to have a benign course, presenting many diagnostic and therapeutic challenges with radiological and clinical characteristics similar to those of other CNS tumors. There is currently no clear evidence of the pathogenesis and therapeutic management of this condition. Follow-up in these patients will help elucidate the natural history of this condition and the benefits of various treatment modalities.

\section{References}

1. Abla O, Jacobsen E, Picarsic J, et al. Consensus recommendations for the diagnosis and clinical management of Rosai-DorfmanDestombes disease. Blood. 2018;131(26):2877-2890.

2. Taufiq M, Khair A, Begum F, et al. Isolated intracranial RosaiDorfman disease. Case Rep Neurol Med. 2016;2016:1972594.

3. Triana-Pérez AB, Sánchez-Medina Y, Pérez-Del Rosario PA, et al. Isolated intracranial Rosai-Dorfman disease: a case report and literature review. Article in Spanish. Neurocirugia (Astur). 2011;22(3):255-260.

4. Jurić G, Jakić-Razumović J, Rotim K, Zarković K. Extranodal sinus histiocytosis (Rosai-Dorfman disease) of the brain parenchyma. Acta Neurochir (Wien). 2003;145(2):145-149.

5. Lu D, Estalilla OC, Manning JT Jr, Medeiros LJ. Sinus histiocytosis with massive lymphadenopathy and malignant lymphoma involving 
the same lymph node: a report of four cases and review of the literature. Mod Pathol. 2000;13(4):414-419.

6. Foucar E, Rosai J, Dorfman R. Sinus histiocytosis with massive lymphadenopathy (Rosai-Dorfman disease): review of the entity. Semin Diagn Pathol. 1990;7(1):19-73.

7. Andriko JA, Morrison A, Colegial $\mathrm{CH}$, et al. Rosai-Dorfman disease isolated to the central nervous system: a report of 11 cases. Mod Pathol. 2001;14(3):172-178.

8. Toh $\mathrm{CH}$, Chen YL, Wong HF, et al. Rosai-Dorfman disease with dural sinus invasion. Report of two cases. J Neurosurg. 2005; 102(3):550-554.

9. Adeleye AO, Amir G, Fraifeld S, et al. Diagnosis and management of Rosai-Dorfman disease involving the central nervous system. Neurol Res. 2010;32(6):572-578.

10. Zhang Y, Liu J, Zhu J, et al. Case report: Rosai-Dorfman disease involving sellar region in a pediatric patient: a case report and systematic review of literature. Front Med (Lausanne). 2020;7:613756

11. Sandoval-Sus JD, Sandoval-Leon AC, Chapman JR, et al. RosaiDorfman disease of the central nervous system: report of 6 cases and review of the literature. Medicine (Baltimore). 2014;93(3):165-175.

12. Nalini A, Jitender S, Anantaram G, Santosh V. Rosai Dorfman disease: case with extensive dural involvement and cerebrospinal fluid pleocytosis. J Neurol Sci. 2012;314(1-2):152-154.

13. Chen $\mathrm{HH}$, Zhou $\mathrm{SH}$, Wang $\mathrm{SQ}$, et al. Factors associated with recurrence and therapeutic strategies for sinonasal Rosai-Dorfman disease. Head Neck. 2012;34(10):1504-1513.

14. Goupil de Bouillé J, de Muret A, Diot E, et al. Pulmonary manifestations revealing Rosai-Dorfman disease. Sarcoidosis Vasc Diffuse Lung Dis. 2015;32(3):275-277.

15. Krishnan A, Nassar A, Nieh PT. Rosai-Dorfman disease presenting as extranodal renal mass. Urology. 2005;66(6):1319.

16. Albano D, Bosio G, Bertagna F. ${ }^{18}$ F-FDG PET/CT follow-up of Rosai-Dorfman disease. Clin Nucl Med. 2015;40(8):e420-e422.

17. Pulsoni A, Anghel G, Falcucci $P$, et al. Treatment of sinus histiocytosis with massive lymphadenopathy (Rosai-Dorfman disease): report of a case and literature review. Am J Hematol. 2002;69(1):67-71.

18. Forest $F$, N'guyen AT, Fesselet J, et al. Meningeal Rosai-Dorfman disease mimicking meningioma. Ann Hematol. 2014;93(6):937-940.

19. Z'Graggen WJ, Sturzenegger M, Mariani L, et al. Isolated RosaiDorfman disease of intracranial meninges. Pathol Res Pract. 2006;202(3):165-170.
20. McPherson CM, Brown J, Kim AW, DeMonte F. Regression of intracranial Rosai-Dorfman disease following corticosteroid therapy. Case report. J Neurosurg. 2006;104(5):840-844.

21. Rivera $D$, Pérez-Castillo $M$, Fernández $B$, Stoeter P. Long-term follow-up in two cases of intracranial Rosai-Dorfman disease complicated by incomplete resection and recurrence. Surg Neurol Int. 2014;5:30.

22. Maia RC, de Meis E, Romano S, et al. Rosai-Dorfman disease: a report of eight cases in a tertiary care center and a review of the literature. Braz J Med Biol Res. 2015;48(1):6-12.

23. Maklad AM, Bayoumi Y, Tunio M, et al. Steroid-resistant extranodal Rosai-Dorfman disease of cheek mass and ptosis treated with radiation therapy. Case Rep Hematol. 2013;2013:428297.

24. Sasidharan A, Verma A, Epari S, et al. Symptomatic intracranial Rosai-Dorfman disease in the suprasellar region treated with conformal radiotherapy: a report of two cases and literature review. Neurol India. 2020;68(2):489-492.

25. Boissaud-Cooke MA, Bhatt K, Hilton DA, Muquit S. Isolated intracranial Rosai-Dorfman disease: case report and review of the literature. World Neurosurg. 2020;137:239-242.

26. Wang W, Sun J, Zhang W, Zhou D. Successful treatment of intracranial Rosai-Dorfman disease with cytarabine and dexamethasone: case report and review of literature. Ann Hematol. 2020;99(5):1157-1159.

\section{Disclosures}

The authors report no conflict of interest concerning the materials or methods used in this study or the findings specified in this paper.

\section{Author Contributions}

Conception and design: all authors. Acquisition of data: Wong-Achi, Apolo. Analysis and interpretation of data: Wong-Achi. Drafting the article: all authors. Critically revising the article: Wong-Achi, Chong. Reviewed submitted version of manuscript: all authors. Approved the final version of the manuscript on behalf of all authors: Wong-Achi. Statistical analysis: Wong-Achi. Administrative/technical/material support: all authors. Study supervision: Wong-Achi, Chong.

\section{Correspondence}

Xavier Wong-Achi: Universidad Espíritu Santo, Samborondón, Ecuador. xawong@uees.edu.ec. 